\title{
Rotational spectroscopy and dipole moment of cis-cis HOONO and DOONO
}

\author{
Juliane L. Frya) \\ Arthur Amos Noyes Laboratory of Chemical Physics, California Institute of Technology, Pasadena, \\ California 91125
}

Brian J. Drouin ${ }^{\text {b) }}$ and Charles E. Miller

Jet Propulsion Laboratory, California Institute of Technology, Pasadena, California 91109

(Received 11 October 2005; accepted 8 December 2005; published online 22 February 2006)

\begin{abstract}
The rotational spectrum of cis-cis HOONO has been studied over a broad range of frequencies, 13-840 GHz, using pulsed beam Fourier-transform microwave spectroscopy and room-temperature flow cell submillimeter spectroscopy. The rotational spectrum of the deuterated isotopomer, cis-cis DOONO, has been studied over a subset of this range, 84-640 GHz. Improved spectroscopic constants have been determined for HOONO, and the DOONO spectrum is analyzed for the first time. Weak-field Stark effect measurements in the region of $84-110 \mathrm{GHz}$ have been employed to determine the molecular dipole moments of cis-cis HOONO $\left[\mu_{a}=0.542(8) \mathrm{D}, \mu_{b}\right.$ $=0.918(15) \mathrm{D}, \mu=1.07(2) \mathrm{D}]$ and DOONO $\left[\mu_{a}=0.517(9) \mathrm{D}, \mu_{b}=0.930(15) \mathrm{D}, \mu=1.06(2) \mathrm{D}\right]$. The quadrupole coupling tensor in the principal inertial axis system for the ${ }^{14} \mathrm{~N}$ nucleus has been determined to be $\chi_{a a}=1.4907(25) \mathrm{MHz}, \chi_{b b}=-4.5990(59) \mathrm{MHz}, \chi_{a b}=3.17(147) \mathrm{MHz}$, and $\chi_{c c}$ $=3.1082(59) \mathrm{MHz}$. Coordinates of the $\mathrm{H}$ atom in the center-of-mass frame have been determined with use of the Kraitchman equations, $\left|a_{\mathrm{H}}\right|=0.516 \AA$ and $\left|b_{\mathrm{H}}\right|=1.171 \AA$. The inertial defects of HOONO and DOONO are consistent with a planar equilibrium structure with significant out-of-plane $\mathrm{H}$ atom torsional motion. Comparisons of the present results are made to ab initio calculations. @ 2006 American Institute of Physics. [DOI: 10.1063/1.2163341]
\end{abstract}

\section{INTRODUCTION}

The primary loss mechanism for $\mathrm{HO}_{x}\left(\mathrm{OH}+\mathrm{HO}_{2}\right)$ and $\mathrm{NO}_{x}\left(\mathrm{NO}+\mathrm{NO}_{2}\right)$ radicals in the atmosphere is the three-body radical-radical association reaction,

$$
\mathrm{OH}+\mathrm{NO}_{2}+M \rightarrow \mathrm{HNO}_{3}+M
$$

Knowledge of the rate constant for this reaction is critical to accurate modeling of atmospheric chemistry since catalytic $\mathrm{HO}_{x}$ and $\mathrm{NO}_{x}$ reaction cycles regulate ozone concentrations in the upper troposphere and stratosphere, as well as ozone formation and air quality in the lower troposphere. Highlevel $a b$ initio calculations have identified cis-cis and transperp peroxynitrous acid as stable minima on the HOONO potential-energy surface. ${ }^{1-5}$ Experimental measurements of reaction (1) are thus complicated by the existence of multiple $\mathrm{HNO}_{3}$ products,

$$
\begin{aligned}
\mathrm{OH}+\mathrm{NO}_{2}+M & \rightarrow \mathrm{HONO}_{2}+M \\
& \rightarrow \text { cis-cis } \mathrm{HOONO}+M \\
& \rightarrow \text { trans-perp } \mathrm{HOONO}+M .
\end{aligned}
$$

The formation of the stable nitric acid isomer, $D_{0}\left(\mathrm{HO}-\mathrm{NO}_{2}\right) \sim 200 \mathrm{~kJ} / \mathrm{mol}$, via reaction (1a) sequesters $\mathrm{OH}$ and $\mathrm{NO}_{2}$ radicals in a nonreactive reservoir species. Alternatively, formation of the less stable HOONO isomer,

\footnotetext{
${ }^{a}$ Electronic mail: fry@caltech.edu

b) Author to whom correspondence should be addressed. Electronic mail: brian.j.drouin@jpl.nasa.gov
}

$D_{0}(\mathrm{HO}-\mathrm{ONO}) \sim 80 \mathrm{~kJ} / \mathrm{mol}$, via reactions $(1 \mathrm{~b})$ and $(1 \mathrm{c})$, regenerates the $\mathrm{OH}$ and $\mathrm{NO}_{2}$ radicals due to rapid thermal decomposition. In fact, cis-cis HOONO measurements provide an effective proxy for the total HOONO yield, (1b) and (1c), under atmospheric conditions since a recent kinetics study demonstrated that the trans-perp conformer isomerizes quickly to the more stable cis-cis HOONO (Fig. 1) at atmospherically relevant temperatures. ${ }^{6}$

Recent kinetics and spectroscopy experiments have found both the direct and indirect evidence for HOONO production in the $\mathrm{OH}+\mathrm{NO}_{2}$ reaction, ${ }^{2,6-8}$ and the vibrational spectroscopy of HOONO is a subject of continued study. ${ }^{9-12}$ The current best estimate of the $\mathrm{HOONO} / \mathrm{HONO}_{2}$ branching ratio is $0.075 \pm 0.020(2 \sigma)$ at $295 \mathrm{~K}$ and 20 Torr. $^{2}$ Recent spectroscopic modeling suggests an upward adjustment of this branching ratio by approximately $25 \% .^{13}$ Ongoing experiments to determine the temperature and pressure depen-

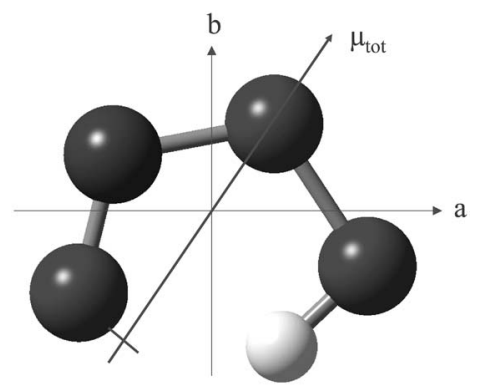

FIG. 1. Calculated structure of cis-cis HOONO in principal axis system. The molecule is planar, with partial $\mathrm{H}$ bonding between the terminal $\mathrm{O}$ and $\mathrm{H}$ atoms. The total dipole moment vector is shown. 


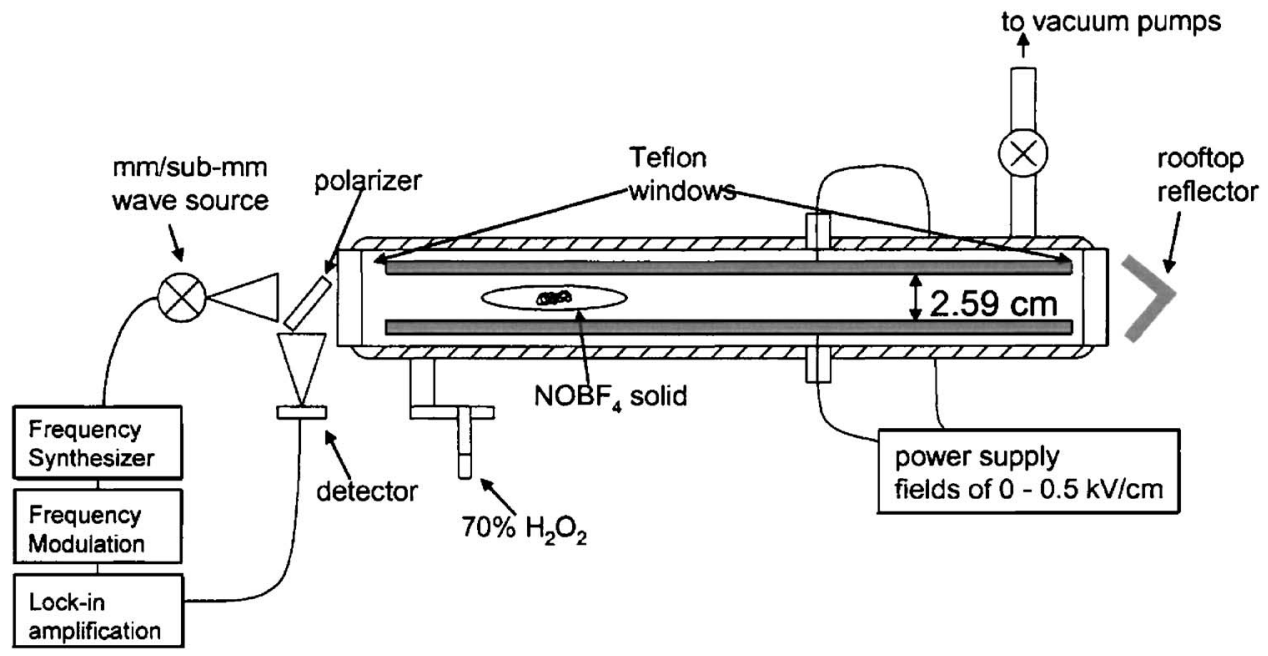

FIG. 2. Schematic of JPL submillimeter experimental apparatus. Microwave radiation makes two passes through the cell, with $90^{\circ}$ polarization rotation due to the rooftop reflector. Stark plates are at a separation of $2.594(44) \mathrm{cm}$, and fields of $0-1.25 \mathrm{kV}$ are generated across them. $\mathrm{NOBF}_{4}$ solid is placed in boat in the interior of the cell, over which the $70 \% \mathrm{H}_{2} \mathrm{O}_{2}$ flows.

dences of the branching ratio ${ }^{14}$ suggest a larger $\mathrm{HOONO} / \mathrm{HONO}_{2}$ branching ratio under atmospheric conditions, consistent with kinetic modeling predictions. ${ }^{5}$ All experimental evidence point to a significant rate of HOONO formation in the atmosphere, but it has not been detected in the atmosphere to date.

Our previous work ${ }^{15}$ on the pure rotation spectrum of cis-cis HOONO provided a potential means for specific atmospheric identification from balloon, aircraft, or groundbased millimeter and submillimeter spectrometers. An accurate determination of the permanent electric dipole moment is essential to determine the measurement sensitivity required for atmospheric detection or to quantify HOONO concentrations measured in absorption or emission in the laboratory. We have measured Stark shifts of carefully chosen low- $J$ transitions to obtain $\mu_{a}, \mu_{b}$, and $\mu_{\text {total }}$. The results are compared to spectral simulations and $a b$ initio calculations for the vibrationally averaged zero-point level. Additionally, we report the rotation spectrum and dipole moment of DOONO as well as the substitution coordinates of the hydrogen atom. These center-of-mass coordinates render the molecular assignment of the spectrum completely unambiguous, and allow comparison with $a b$ initio structure calculations.

\section{EXPERIMENTAL METHODS}

The millimeter and submillimeter measurements were carried out at JPL using a 1-m-long glass cell in double-pass configuration, with sources and detectors at one end of the cell and a polarization-rotating rooftop reflector at the opposite end (Fig. 2). The source and detector are mounted at a relative angle of $90^{\circ}$, with a polarized grid at $45^{\circ}$ to pass from the source and reflect to the detector. Specifics of the multipliers and detectors in use on the millimeter and submillimeter spectrometer are given elsewhere. ${ }^{16}$ For the Stark effect measurements, two parallel-plate electrodes are inserted into the flow cell at an effective separation of $d$ $=2.594(44) \mathrm{cm}$.

HOONO was generated by reaction of gaseous $70 \%$ $\mathrm{H}_{2} \mathrm{O}_{2}$ in $\mathrm{H}_{2} \mathrm{O}$ (FMC Corporation) with solid $\mathrm{NOBF}_{4}$ (Aldrich). The $\mathrm{NOBF}_{4}$ solid was placed in a shallow Pyrex boat inside the absorption cell, over which the hydrogen peroxide vapor flowed from a sidearm port. A slow flow achieved through constriction at the pump port allowed the longest duration of HOONO production. Faster flows reduced the amount of observable by-products (primarily $\mathrm{HONO}_{2}$, but also $\mathrm{HO}_{2} \mathrm{NO}_{2}$ and $\left.\mathrm{BF}_{2} \mathrm{OH}\right)$ but increased the required frequency of venting the system to refill the boat. $\mathrm{D}_{2} \mathrm{O}_{2}$ (ICON Isotopes), $\sim 50 \%$ in $\mathrm{D}_{2} \mathrm{O}$, was substituted for $\mathrm{H}_{2} \mathrm{O}_{2}$ as the precursor for DOONO. The cell was conditioned with $\mathrm{D}_{2} \mathrm{O}$ prior to the DOONO search and discovery. Substantial HOONO was still observable in the deuterated system, perhaps an indication of atmospheric water absorbed in the hygroscopic $\mathrm{NOBF}_{4}$ sample. In all cases, the vacuum pump valve was adjusted to optimize the flow rate of $\mathrm{H}_{2} \mathrm{O}_{2}\left(\mathrm{D}_{2} \mathrm{O}_{2}\right)$ for maximum HOONO (DOONO) production.

The low-frequency rotational spectra of HOONO were collected in a supersonic expansion inside the Mark II Flygare-Balle Fourier-transform microwave spectrometer at Caltech. ${ }^{17}$ In these experiments, the $\mathrm{NOBF}_{4}$ solid is contained behind a wire mesh in a cavity next to the channel of the pulsed valve housing, directly downstream of the poppet. This solid sample holder was designed for the heating of low-volatility solid samples, ${ }^{18}$ but was used in these experiments at room temperature only. Argon carrier gas is bubbled through a $70 \% \mathrm{H}_{2} \mathrm{O}_{2}$ solution to entrain the reactant $\mathrm{H}_{2} \mathrm{O}_{2}$ in the gas phase, and subsequently pulsed through the sample holder and into the Fourier-transform microwave (FTMW) chamber.

\section{RESULTS AND DISCUSSION}

\section{A. Observed spectra and assignment}

All spectral fitting and rotational line simulations were performed using Pickett's SPFIT program suite. ${ }^{19}$ The DOONO spectrum was predicted by isotopic shifting of the HOONO spectrum. Characteristic spectral signatures were identified within a few hundreds of megahertz of the estimated positions. These transitions were fitted to refine the prediction and the search continued. The transition frequencies were well predicted after two to three iterations of this process.

The submillimeter wave rotational spectroscopy of ciscis HOONO has been expanded from the previous assign- 
TABLE I. Fitted cis-cis HOONO and DOONO Hamiltonian parameters. Values in parentheses represent $1 \sigma$ uncertainties in units of the least significant figure. Transitions below 13-19 GHz have uncertainty of $2 \mathrm{kHz}, 20$ $122 \mathrm{GHz}$ have uncertainty of $40 \mathrm{kHz}, 123-640 \mathrm{GHz}$ have uncertainty of 50 $\mathrm{kHz}$, and $641-847 \mathrm{GHz}$ have uncertainty of $70 \mathrm{kHz}$.

\begin{tabular}{llcc}
\hline \hline Parameter & Units & HOONO & DOONO \\
\hline$A$ & $\mathrm{MHz}$ & $21406.39252(61)$ & $20245.01569(157)$ \\
$B$ & $\mathrm{MHz}$ & $7948.05526(32)$ & $7913.26701(72)$ \\
$C$ & $\mathrm{MHz}$ & $5791.00077(28)$ & $5685.34813(63)$ \\
$-\Delta_{J}$ & $\mathrm{kHz}$ & $-8.053692(223)$ & $-8.08964(104)$ \\
$-\Delta_{J K}$ & $\mathrm{kHz}$ & $16.03416(99)$ & $13.8573(49)$ \\
$-\Delta_{K}$ & $\mathrm{kHz}$ & $-59.98412(32)$ & $-47.067(38)$ \\
$-\delta_{J}$ & $\mathrm{kHz}$ & $-2.472485(77)$ & $-2.529099(257)$ \\
$-\delta_{K}$ & $\mathrm{kHz}$ & $-13.73624(181)$ & $-13.3029(39)$ \\
$\Phi_{J}$ & $\mathrm{mHz}$ & $-14.913(60)$ & $-15.36(44)$ \\
$\Phi_{J J K}$ & $\mathrm{mHz}$ & $185.55(50)$ & $165.4(50)$ \\
$\Phi_{J K K}$ & $\mathrm{mHz}$ & $-888.16(189)$ & $-729.7(202)$ \\
$\Phi_{K}$ & $\mathrm{~Hz}$ & $1.1005(46)$ & $0.729(192)$ \\
$\phi_{J}$ & $\mathrm{mHz}$ & $-5.2803(232)$ & $-5.237(142)$ \\
$\phi_{J K}$ & $\mathrm{mHz}$ & $-53.36(70)$ & $-54.7(36)$ \\
$\phi_{K}$ & $\mathrm{mHz}$ & $397.7(43)$ & $298(35)$ \\
$1.5 \chi_{a a}$ & $\mathrm{MHz}$ & $2.2361(37)$ & $1.498(283)$ \\
$1 / 4\left(\chi_{b b}-\chi_{c c}\right)$ & $\mathrm{MHz}$ & $-1.9268(15)$ & $-1.9163(277)$ \\
$\chi_{a b}$ & $\mathrm{MHz}$ & $3.17(147)$ & $3.17^{\mathrm{a}}$ \\
$\sigma_{\text {fit }} /$ no. of lines & $\mathrm{kHz}$ & $50.6 / 480$ & $47.5 / 246$ \\
\hline \hline
\end{tabular}

${ }^{\mathrm{a}}$ Value fixed to that determined for HOONO.

ment of 225 lines in the $179-640 \mathrm{GHz}$ range, fit with a standard deviation of $48.4 \mathrm{kHz},{ }^{15}$ to a current data set of 480 lines (115 blended lines of 2, 3, or 4 transitions and 174 nonblended lines) in the $13-840 \mathrm{GHz}$ range, fit with a standard deviation of $50.6 \mathrm{kHz}$. The measured and calculated frequencies and quantum number assignments are found in the JPL millimeter and submillimeter spectral line catalog at http://spec.jpl.nasa.gov/catalog, and the HOONO SPFIT and SPCAT files are included in the supplemental material. ${ }^{20}$ Rotational constants and distortion constants determined from the least-squares fit to this extended data set are shown in Table I.

Cis-cis DOONO scans in the range of $80-640 \mathrm{GHz}$ resulted in a data set of 246 lines (54 blended lines of 2, 3, or 4 transitions and 103 nonblended lines), up to $J=42$ and $K_{a}=18$ fit with a standard deviation of $47.5 \mathrm{kHz}$. The measured and calculated frequencies and quantum number assignments of DOONO are also available in the supplemental material in the form of SPCAT and SPFIT files. ${ }^{20}$ Spectroscopic constants determined from the least-squares fit to this data set are also shown in Table I.

Figure 3 shows the measured and simulated spectra of HOONO and DOONO near $270 \mathrm{GHz}$. In the $K_{c}=21-20 \mathrm{R}$ bunch, only the lowest two $K_{a}$ components are still "bunched," with $K_{c}=0,1$ (not shown) collapsed as a degenerate quartet and $K_{c}=1,2$ split into quartets with characteristic intensity patterns. Both panels of Fig. 3 are $20 \mathrm{MHz}$ wide, showing the larger spread of the asymmetry splitting in HOONO. The DOONO quartet is more compact and a completely separated $\mathrm{HONO}_{2}$ feature is visible within the 20 $\mathrm{MHz}$ window. The simulations of each quartet take into account the dipole component measurements, which are differ-
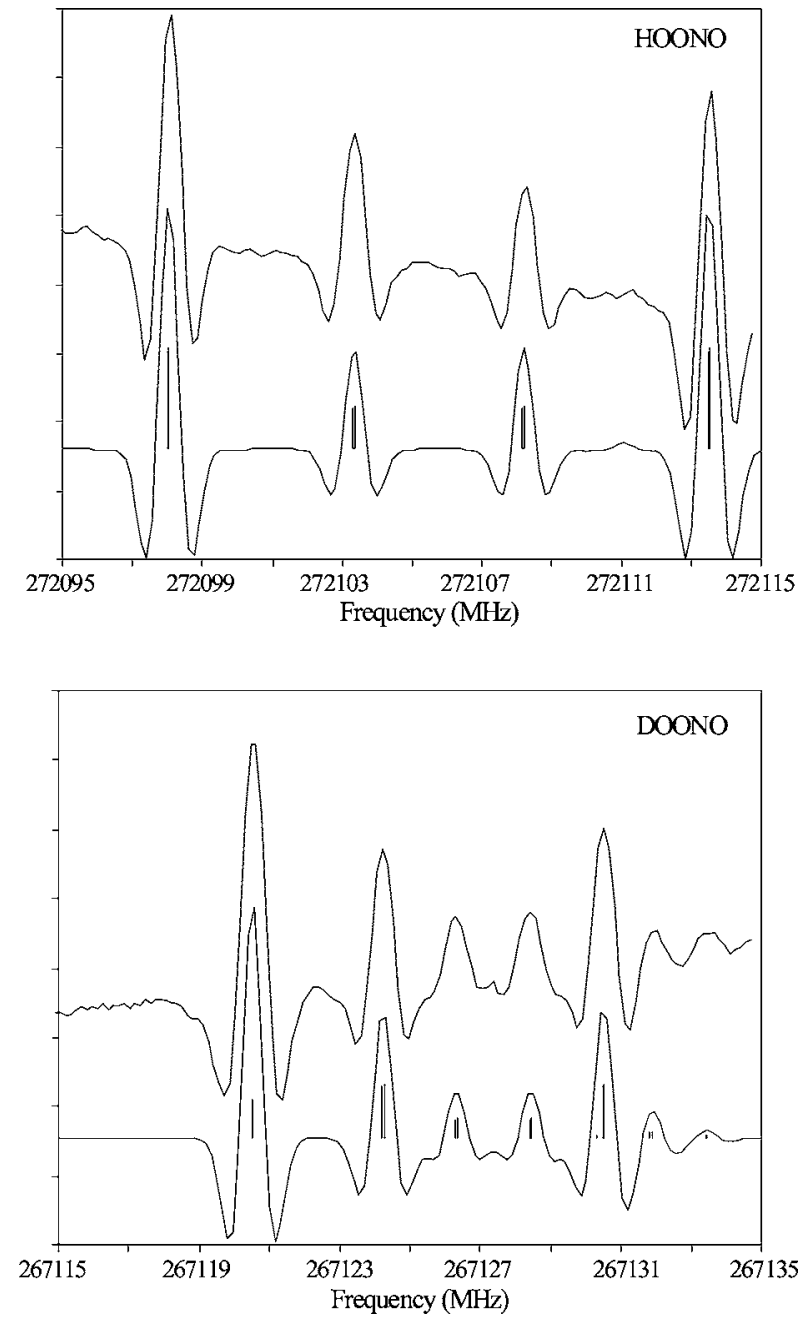

FIG. 3. The $J=22 \leftarrow 21 K_{c}=21 \leftarrow 20$ quartets of HOONO and DOONO. The upper traces are measurement, and the lower traces are simulations $(0.5$ MHz FWHM second derivative Gaussian and stick spectra) of predicted HOONO, DOONO (black), and $\mathrm{HONO}_{2}$ (gray) transitions.

ent for HOONO and DOONO due to a small principal axis rotation. These dipole components are described in the next section and Fig. 3 shows the corresponding change in relative intensities of the inner and outer lines in the quartets.

\section{B. Stark effect measurement of dipole moments}

The dipole moments of both HOONO and DOONO were determined by examining Stark effect shifts of 10 and 12 rotational transitions, respectively, in the region of 84$110 \mathrm{GHz}$ under weak-field conditions $(0 \leqslant \varepsilon \leqslant 0.5 \mathrm{kV} / \mathrm{cm})$. The separation between Stark plates in the spectrometer was found to be $d=2.594(44) \mathrm{cm}$ after calibration (see below). Therefore the maximum applied field strength was $\varepsilon=V / d$ $=482 \mathrm{~V} \mathrm{~cm}^{-1}$.

The weak-field method, described previously by Muller et al. ${ }^{21,22}$ measures the second-order Stark shifts of the intensity weighted averages of unresolved $M_{J}$ Stark components. The shift for an energy level is of the form

$$
\Delta E=\varepsilon^{2}\left[A+B M_{J}^{2}\right],
$$

where $A$ and $B$ are the Stark coefficients and $\varepsilon$ is the strength of the electric field. To calculate the shift for a transition, the 
$\Delta E$ for the two levels are subtracted. For a reliable weakfield measurement, the combined shifts of the individual Stark components of both the upper and lower levels of each of the $M_{J}$ substates must be contained within the linewidth of the measurement. This requirement excludes the use of any near-degenerate energy levels that show rapid splitting of the $M_{J}$ levels due to the first-order Stark effect.

The use of a rooftop reflector to rotate the polarization of the millimeter beam radiation by $90^{\circ}$ before the second pass of the Stark cell ensured that equal contributions from the $\Delta M_{J}=0$ and $\Delta M_{J}= \pm 1$ components were measured. The intensity weighted average of each transition from each $M_{J}$ sublevel is a function of the upper and lower state Stark effect coefficients $A^{\prime}, A^{\prime \prime}, B^{\prime}$ and $B^{\prime \prime}$. This average was calculated for each component, $g(g=a, b)$.

$$
\begin{aligned}
\frac{\Delta \nu_{g}}{\varepsilon^{2}}= & \left(A_{g}^{\prime}-A_{g}^{\prime \prime}\right)+\left(B_{g}^{\prime}-B_{g}^{\prime \prime}\right) \frac{[8 J(J+1)]}{20} \text { for } \Delta J=0, \\
\frac{\Delta \nu_{g}}{\varepsilon^{2}}= & +\left[\left(A_{g}^{\prime}-A_{g}^{\prime \prime}\right)+\left(B_{g}^{\prime}-B_{g}^{\prime \prime}\right) \frac{J(6 J+7)}{20}\right. \\
& \left.+B_{g}^{\prime} \frac{J+1}{2}\right] \text { for } J+1 \leftarrow J \\
\frac{\Delta \nu_{g}}{\varepsilon^{2}}= & -\left[\left(A_{g}^{\prime}-A_{g}^{\prime \prime}\right)+\left(B_{g}^{\prime}-B_{g}^{\prime \prime}\right) \frac{J(6 J+7)}{20}\right. \\
& \left.+B_{g}^{\prime \prime} \frac{J+1}{2}\right] \quad \text { for } J \leftarrow J+1 .
\end{aligned}
$$

The Stark field strength, $\varepsilon$, was calibrated to account for plate separation as well as inhomogeneity in the overlap of the quasioptical millimeter-wave beam and the electric field by measuring several Stark shifts for $\mathrm{SO}_{2}[\mu$ $=1.63305(4) \mathrm{D}] .^{23}$ Stark shifts were measured for ten $\mathrm{SO}_{2}$ transitions to yield a corrected plate separation that also inherently calibrated any linear error in the voltage measurement. The transitions were chosen to fall in the same frequency range as those measured for HOONO; this eliminated the need to consider any frequency dependence in the calibration. The calibration factor is $d=2.599(44) \mathrm{cm}$, yielding measurements with $1.7 \%$ accuracy at the $1 \sigma$ level.

The HOONO and DOONO lines were chosen for Stark shift measurements based on predicted signal strength, shifted line shape, and Stark coefficients. A complete list of the $10 \mathrm{HOONO}$ and 12 DOONO lines used for the Stark analysis is given in Table II, with quantum numbers and zero-field frequencies listed for each line. Two different statistical procedures were used to analyze the data to ensure proper treatment of the measurement statistics. Both procedures began with measurements of line center and shifts of the line center as a function of the applied voltage. The zerofield line center was measured before and after measurements with the applied field and the average frequency was used as the origin for the shift calculation. These before and after zero-field measurements differed from each other by less than $15 \mathrm{kHz}$ for each line. In the first statistical method,

\begin{tabular}{|c|c|c|c|}
\hline \multicolumn{4}{|c|}{ Stark effect lines measured } \\
\hline \multicolumn{2}{|c|}{ HOONO } & \multicolumn{2}{|c|}{ DOONO } \\
\hline$J_{K_{a^{\prime}}^{\prime} K_{c^{\prime}}} \leftarrow J_{K_{a}, K_{c}}$ & $\begin{array}{l}\text { Observed frequency } \\
\text { (zero field) }\end{array}$ & $J_{K_{a^{\prime}}^{\prime} K_{c^{\prime}}}^{\prime} \leftarrow J_{K_{a}, K_{c}}$ & $\begin{array}{c}\text { Observed frequency } \\
\text { (zero field) }\end{array}$ \\
\hline $7_{1,7} \leftarrow 6_{0,6}$ & 89113.199 & $7_{1,7} \leftarrow 6_{0,6}$ & 86985.395 \\
\hline $7_{0,7} \leftarrow 6_{1,6}$ & 84732.389 & $8_{1,7} \leftarrow 7_{1,7}$ & 96431.690 \\
\hline $8_{0,8} \leftarrow 7_{1,7}$ & 97226.837 & $8_{1,8} \leftarrow 7_{0,7}$ & 97620.973 \\
\hline $8_{1,8} \leftarrow 7_{0,7}$ & 99787.057 & $8_{0,8} \leftarrow 7_{1,7}$ & 95784.832 \\
\hline $8_{4,5} \leftarrow 8_{3,6}$ & 101548.048 & $9_{1,9} \leftarrow 8_{1,8}$ & 107907.758 \\
\hline $8_{4,4} \leftarrow 8_{3,5}$ & 95377.054 & $9_{4,6} \leftarrow 9_{3,7}$ & 95330.742 \\
\hline $9_{0,9} \leftarrow 8_{1,8}$ & 109353.886 & $9_{4,5} \leftarrow 9_{3,6}$ & 82232.494 \\
\hline $16_{5,11} \leftarrow 16_{4,12}$ & 95637.084 & $18_{5,13} \leftarrow 18_{4,14}$ & 83697.383 \\
\hline $18_{5,13} \leftarrow 18_{4,14}$ & 89262.020 & $20_{5,15} \leftarrow 20_{4,16}$ & 97563.298 \\
\hline \multirow[t]{3}{*}{$20_{5,15} \leftarrow 20_{4,16}$} & 95492.054 & $21_{6,15} \leftarrow 21_{5,16}$ & 97547.901 \\
\hline & & $22_{6,16} \leftarrow 22_{5,17}$ & 98866.419 \\
\hline & & $23_{6,17} \leftarrow 23_{5,18}$ & 104287.942 \\
\hline
\end{tabular}
the Stark shifts for each transition were fit as a function of $\varepsilon^{2}$. Significant deviations from linearity occurred when a
TABLE II. Complete listing of lines for which Stark effect measurements were conducted.

transition passed out of the weak-field limit. Therefore, measurements for several of the strongest shifts were removed from the analysis to ensure that all data were within the weak-field approximation. The largest Stark shifts included in the dipole moment fitting were up to $400 \mathrm{kHz}$ for HOONO and $140 \mathrm{kHz}$ for DOONO. In method I, the $1 / 0$ slopes (12 for DOONO) from each regression for each transition were used in a nonlinear fit to the dipole moment components.

$$
\frac{\Delta \nu}{\varepsilon^{2}}=C_{a} \Delta \nu_{a}+C_{b} \Delta \nu_{b}
$$

Fixed values for $A^{\prime}, A^{\prime \prime}, B^{\prime}$, and $B^{\prime \prime}$ determined with a unitary dipole component (under this condition $C_{g}=\mu_{g}$ ) were used to calculate the $\Delta \nu_{g}$. The coefficients $\left(C_{a}\right.$ and $\left.C_{b}\right)$ representing the magnitude of each dipole moment component were then varied to minimize the sum of the squared differences of the measured and calculated slope divided by its uncertainty. The weighted fit was necessary because the measured slopes range over several orders of magnitude. Examples of the individual Stark shift regression measurements are shown in Fig. 4. All Stark shift data are available in the supplemental material. ${ }^{20}$

To check the method I results, a second independent statistical analysis was performed (method II) in which all 177 measured HOONO Stark shifts (158 measurements for DOONO) were fit simultaneously to the parameters $C_{a}$ and $C_{b}$ using Eq. (5),

$$
\Delta \nu(\varepsilon)=\varepsilon^{2} C_{a} \Delta \nu_{a}+\varepsilon^{2} C_{b} \Delta \nu_{b} .
$$

Both procedures produced precise values for $\mu_{a}$ and $\mu_{b}$ with differences of less than $4 \%$ in $\mu_{a}$ and less than $2 \%$ in $\mu_{b}$. Method II weights all transitions only by their inherent center frequency shift and the quality of this measurement, so this method has been chosen as the preferred approach. The resulting dipole moments are shown in Table III. These components are in good agreement (within $3 \sigma$ ) with ab initio calculations of the vibrationally averaged components of the dipole moment, ${ }^{24}$ also shown in Table III. 



FIG. 4. Selected Stark effect measurements for $a$-type and $b$-type rotational transitions of HOONO and DOONO.

The direction of the electric dipole moment in HOONO can be determined unambiguously from a comparison of the differences in the axis rotation that is apparent from the $\mu_{a}$ and $\mu_{b}$ components of the dipole moment for HOONO and DOONO. When the principal axis are defined to place the nitrogen atom in the $(-a,+b)$ quadrant and hydrogen in the $(+a,-b)$ quadrant (as in Fig. 1), the negative pole of the dipole vector is oriented towards the $(+a,+b)$ quadrant. This orientation is expected since the $\mathrm{H}-\mathrm{O}$ bond has the largest

TABLE III. Dipole moments (Debye) for cis-cis HOONO and DOONO determined by Stark effect analysis. Values in parentheses are $1 \sigma$ accuracy in the last significant figure derived from the calibration.

\begin{tabular}{llllll}
\hline \hline & \multicolumn{2}{c}{ HOONO } & & \multicolumn{2}{c}{ DOONO } \\
\cline { 2 - 3 } \cline { 5 - 6 } & Experiment & Ab initio $^{\mathrm{a}}$ & & Experiment & Ab initio $^{\mathrm{a}}$ \\
\hline$\mu_{a}$ & $0.542(8)$ & 0.531 & & $0.517(9)$ & 0.520 \\
$\mu_{b}$ & $0.918(15)$ & 0.869 & & $0.930(15)$ & 0.874 \\
$\mu_{\text {total }}$ & $1.07(2)$ & 1.018 & & $1.06(2)$ & 1.016 \\
$N_{\text {lines }}$ & 10 & & & 12 & \\
\hline \hline
\end{tabular}

${ }^{\mathrm{a}} \mathrm{Ab}$ initio dipole moment components are from the vibrationally averaged CCSD(T)/ANO calculations by Zhang et al. (Ref. 24) and Stanton (Ref. 28).
TABLE IV. Cis-cis HOONO and DOONO moments of inertia and inertial defects. Values in parentheses are $1 \sigma$ uncertainties based on the fitted parameters. Correlations among the parameters are minimal and were ignored.

\begin{tabular}{lcc}
\hline \hline & HOONO & DOONO \\
\hline$I_{a a}\left(\mathrm{amu} \AA^{2}\right)$ & $23.6087918(7)$ & $24.963135(2)$ \\
$I_{b b}\left(\mathrm{amu} \AA^{2}\right)$ & $63.585248(3)$ & $63.864781(6)$ \\
$I_{c c}\left(\mathrm{amu} \AA^{2}\right)$ & $87.269728(4)$ & $88.891489(10)$ \\
$\Delta\left(\mathrm{amu} \AA^{2}\right)$ & $0.075688865(5)$ & $0.063573434(10)$ \\
\hline \hline
\end{tabular}

electronegativity differential in the molecule, and this bond is roughly parallel to the total dipole moment vector (see Fig. $1)$. The $1.7 \%$ uncertainty $(1 \sigma)$ in the calibration factor $d$ dominates the overall accuracy of the dipole moment determination.

\section{Position of $\mathrm{H}$ atom in principal axis frame}

The rotational constants for HOONO and DOONO reported in Table I have been converted into moments of inertia, shown in Table IV. The moments of inertia have also been used to determine the substitution coordinates of the hydrogen atom in the principal axis center-of-mass frame of the molecule using the well-known Kraitchman equations. $^{25,26}$ The experimentally determined center-ofmass $\mathrm{H}$ atom coordinates are reported in Table $\mathrm{V}$.

The structural parameters calculated in this way represent a ground-state effective structure $\left(r_{s}\right)$, which includes any vibrational effects in the ground-state moments of inertia used to determine the structural parameters. This is evidenced by the nonzero out-of-plane root-mean-squared (rms) displacement of the $\mathrm{H}$ atom position. In spite of the planarity of cis-cis HOONO in the $r_{e}$ structure, zero-point torsional motion results in a nonzero $\mathrm{rms}$ displacement of the $\mathrm{H}$ atom out of plane.

This experimental $\mathrm{H}$ atom position is compared to the $a b$ initio calculated $\mathrm{H}$ atom position from several calculations in Table V. The ground-state structure of cis-cis HOONO was first calculated by McGrath and Rowland at MP2/6-311G $(d, p),{ }^{1}$ and later by Tsai et al. at CCSD/6$311++\mathrm{G}(d, p),{ }^{27}$ by Fry et al. at $\mathrm{CCSD}(\mathrm{T}) / \mathrm{cc}-\mathrm{pVTZ},{ }^{6}$ and by Stanton at $\operatorname{CCSD}(\mathrm{T})$ atomic natural orbital (ANO). ${ }^{28}$ This comparison supports the conclusion that high-level CCSD(T) calculations accurately predict the structural parameters of HOONO.

TABLE V. Comparison of $\mathrm{HOONO}$ experimental and ab initio $\mathrm{H}$ atom coordinates.

\begin{tabular}{llll}
\hline \hline & $|a|$ & $|b|$ & $|c|$ \\
\hline${\text { MP } 2 / 6-311 ~ G(d, p)^{\mathrm{a}}}_{\mathrm{CCSD} / 6-311++\mathrm{G}(d, p)^{\mathrm{b}}}$ & 0.637 & 1.269 & 0.000 \\
$\mathrm{CCSD}(\mathrm{T}) / \mathrm{cc}^{\mathrm{p}} \mathrm{pVTZ}$ & 0.597 & 1.183 & 0.000 \\
$\mathrm{CCSD}(\mathrm{T}) / \mathrm{ANO}^{\mathrm{d}}$ & 0.590 & 1.161 & 0.000 \\
Experiment $^{\mathrm{c}}$ & 0.535 & 1.159 & 0.000 \\
\hline \hline
\end{tabular}

${ }^{\mathrm{a}}$ Reference 1.

${ }^{\mathrm{b}}$ Reference 27.

${ }^{\mathrm{c}}$ Reference 6.

${ }^{\mathrm{d}}$ Reference 28. 


\section{Inertial defect}

The out-of-plane rms displacement of each atom results in a nonzero inertial defect, shown for HOONO and DOONO in Table IV. In HOONO, the positive inertial defect decreases upon deuteration. In a number of planar species $\left(\mathrm{H}_{2} \mathrm{O}, \mathrm{H}_{2} \mathrm{~S}, \mathrm{H}_{2} \mathrm{Se}\right.$, and $\left.\mathrm{H}_{2} \mathrm{CO}\right)$ deuterium substitution increases the (nominally positive) inertial defect due to dominance of in-plane vibrational motions. ${ }^{25,26}$ However, the inertial defect is a balance of in-plane (typically positive) and out-of-plane (typically negative) contributions. The inertial defect for HOONO is smaller than that for $\mathrm{HONO}_{2},{ }^{29}$ indicating more contribution in HOONO from all out-of-plane motions. Cox and Riveros ${ }^{29}$ measured inertial defects for a series of nitric acid isotopomers and found the largest change in inertial defect $\left(-0.0089\right.$ amu $\left.\AA^{2}\right)$ with deuterium substitution. This decrease in inertial defect for $\mathrm{HONO}_{2}$ to $\mathrm{DONO}_{2}$ indicates dominance of $\mathrm{H}$ atom out-of-plane motion, relative to other atoms. The same phenomenon applies in HOONO to DOONO, i.e., the large decrease in inertial defect for deuterium substitution $\left(-0.0121 \mathrm{amu} \AA^{2}\right)$ is consistent with larger amplitude out-of-plane motion of hydrogen than in $\mathrm{HONO}_{2}$.

Calculations of the inertial defects from the $a b$ initio force field ${ }^{28,30}[\Delta(\mathrm{HOONO})=0.0954, \Delta(\mathrm{DOONO})=0.0840$ amu $\left.\AA^{2}\right]$ show similar decrease with deuterium substitution $\left(0.01141 \mathrm{amu} \AA^{2}\right)$ to that observed experimentally. The positive calculated inertial defect indicates the overall dominance of harmonic force field contributions (in plane), while the change in inertial defect upon deuterium substitution is due mainly to the Coriolis (out of plane) components.

\section{E. Nuclear quadrupole coupling constants}

The $I=1$ spin of the ${ }^{14} \mathrm{~N}$ nucleus allowed us to measure nuclear quadrupole splitting on selected transitions. Many of the millimeter wavelength transitions of both HOONO and DOONO with $J<25$ show characteristic quadrupole splitting patterns. The splittings in $Q$-branch transitions are resolved enough to determine $\chi_{b b}-\chi_{c c}$ with four significant figures. Furthermore, the high precision measurements attained in the Flygare-Balle Fourier-transform microwave spectrometer helped improve the precision of the nuclear quadrupole coupling parameter, $\chi_{a a}$ for HOONO.

HOONO has $C_{s}$ symmetry in its ground vibrational state, with the average position of each atom lying in the $a b$ plane, leading by symmetry to $\chi_{b c}=\chi_{a c}=0$, but $\chi_{a b} \neq 0$. In general, the weak coupling of ${ }^{14} \mathrm{~N}$ nuclear spin to the rotation of the molecule precludes determination of the full nuclear spinrotation coupling tensor. However, for cis-cis HOONO the $4_{14}$ and $3_{22}$ energy levels are accidentally nearly degenerate and the off-diagonal quadrupole coupling allows perturbations among the $F=2,3$ components. A single measured spectral feature, the $3_{22} \leftarrow 2_{11}$ transition in the millimeter range, samples this perturbation and has been used to determine $\chi_{a b}$. Fits which include the $\chi_{a b}$ term but do not include the $3_{22} \leftarrow 2_{11}$ transition experience a divergence of the fit until a completely undetermined $\chi_{a b}$ value is obtained. The precision of the splitting in this transition dominates the uncertainty in the determination of the principal quadrupole tensor and the determination of the offset angle $|\theta|=23^{\circ}$
$(+5,-8)$, between the principal inertial axis system and the principal quadrupole axes system. Due to the planar symmetry of this species, this angle necessarily lies in the $a b$ plane; however, its sign is not unambiguous without isotopic information.

\section{CONCLUSIONS}

This work extends our previous analysis of the rotational spectrum of cis-cis HOONO. Rotational spectra for HOONO have been measured over frequencies that include nearly the entire range of thermally populated energy levels. The $\mu_{a}$ and $\mu_{b}$ components of the dipole moment have been determined to an accuracy of 3\% using a weak-field Stark shift analysis. Low-frequency, low- $J$ measurements have allowed determination of hyperfine splittings in the rotational spectra due to ${ }^{14} \mathrm{~N}$ nuclear quadrupole coupling. These new data enable the search for cis-cis HOONO via atmospheric remote sensing.

Additionally, the use of deuterated reactants has enabled us to identify and analyze the rotational spectrum of DOONO for the first time. These measurements, combined with our HOONO analyses, provide the precise determination of the hydrogen atom coordinates in the principle axis system. A comparison of HOONO and DOONO dipole moments yields the unambiguous determination of the dipole moment vector. Comparisons between experimental and $a b$ initio dipole moments and $\mathrm{H}$ atom position in the principal axis frame agree well, indicating that high-level theory accurately characterizes cis-cis HOONO. Analysis of the inertial defect for HOONO and DOONO is consistent with a planar equilibrium structure with significant out-of-plane $\mathrm{H}$ atom torsional motion.

\section{ACKNOWLEDGMENTS}

This research was performed in part at the Jet Propulsion Laboratory (JPL), California Institute of Technology, under contract with the National Aeronautics and Space Administration. The flow cell research was supported in part by NSF's Atmospheric Chemistry Program (Grant No. ATM0432377), and the FTMW research was supported in part by NSF's Chemistry Program (Grant No. CHE-0415745). One of the authors (J.L.F.) gratefully acknowledges funding provided by an American Association of University Women Dissertation Fellowship. The authors are particularly grateful to Susanna Widicus Weaver for her valuable help with the FTMW spectrometer, and to John Stanton for providing data prior to publication and his helpful comments on this manuscript. The authors also thank Edward Cohen, Geoffrey Blake, Mitchio Okumura, and Paul Wennberg for insightful discussions.

\footnotetext{
${ }^{1}$ M. P. McGrath and F. S. Rowland, J. Phys. Chem. 98, 1061 (1994).

${ }^{2}$ B. D. Bean, A. K. Mollner, S. A. Nizkorodov, G. Nair, M. Okumura, S. P. Sander, K. A. Peterson, and J. S. Francisco, J. Phys. Chem. A 107, 6974 (2003).

${ }^{3}$ K. Doclo and U. Rothlisberger, Chem. Phys. Lett. 297, 205 (1998).

${ }^{4}$ Y. M. Li and J. S. Francisco, J. Chem. Phys. 113, 7976 (2000).

${ }^{5}$ D. M. Golden, J. R. Barker, and L. L. Lohr, J. Phys. Chem. A 107, 11057 (2003).

${ }^{6}$ J. L. Fry, S. A. Nizkorodov, M. Okumura, C. M. Roehl, J. S. Francisco,
} 
and P. O. Wennberg, J. Chem. Phys. 121, 1432 (2004).

${ }^{7}$ N. M. Donahue, R. Mohrschladt, T. J. Dransfield, J. G. Anderson, and M. K. Dubey, J. Phys. Chem. A 105, 1515 (2001).

${ }^{8}$ S. A. Nizkorodov and P. O. Wennberg, J. Phys. Chem. A 106, 855 (2002).

${ }^{9}$ I. M. Konen, E. X. J. Li, T. A. Stephenson, and M. I. Lester, J. Chem. Phys. 123, 204318 (2005)

${ }^{10}$ I. M. Konen, I. B. Pollack, E. X. J. Li, M. I. Lester, M. E. Varner, and J. F. Stanton, J. Chem. Phys. 122, 094320 (2005).

${ }^{11}$ J. Matthews, A. Sinha, and J. S. Francisco, J. Chem. Phys. 120, 10543 (2004).

${ }^{12}$ J. Matthews and A. Sinha, J. Chem. Phys. 122, 104313 (2005).

${ }^{13}$ A. B. McCoy, J. L. Fry, J. S. Francisco, A. K. Mollner, and M. Okumura, J. Chem. Phys. 122, 104311 (2005).

${ }^{14} \mathrm{M}$. Okumura (private communication).

${ }^{15}$ B. J. Drouin, J. L. Fry, and C. E. Miller, J. Chem. Phys. 120, 5505 (2004)

${ }^{16}$ B. J. Drouin, F. W. Maiwald, and J. C. Pearson, Rev. Sci. Instrum. 76, 093113 (2005)

${ }^{17}$ T. J. Balle and W. H. Flygare, Rev. Sci. Instrum. 52, 33 (1981).

${ }^{18}$ S. L. Widicus Weaver, Ph.D. thesis, California Institute of Technology, 2005.

${ }^{19}$ H. M. Pickett, J. Mol. Spectrosc. 148, 371 (1991).

${ }^{20}$ See EPAPS Document No. E-JCPSA6-124-017603 for the HOONO and DOONO frequencies and assignments deposited as SPFIT and SPCAT files. Any questions on the file format should be directed to the corresponding author, Brian Drouin. Also included are all Stark shift data for HOONO and DOONO. This document can be reached via a direct link in the online article's HTML reference section or via the EPAPS homepage (http://www.aip.org/pubservs/epaps.html).
${ }^{21}$ H. S. P. Muller, E. A. Cohen, and D. Christen, J. Chem. Phys. 110, 11865 (1999).

${ }^{22}$ H. S. P. Muller, C. E. Miller, and E. A. Cohen, J. Chem. Phys. 107, 8292 (1997).

${ }^{23}$ D. Patel, D. Margolese, and T. R. Dyke, J. Chem. Phys. 70, 2740 (1979).

${ }^{24}$ The $a b$ initio calculations were performed at the $\operatorname{CCSD}(\mathrm{T})$ level with an atomic natural orbital basis set. Vibrationally averaged components of the dipole moment were calculations via the expression

$$
\left\langle\mu^{i}\right\rangle=\mu_{e}^{i}+\sum_{k} \frac{\partial \mu^{i}}{\partial q_{k}}\left\langle q_{k}\right\rangle+\frac{1}{2} \frac{\partial^{2} \mu^{i}}{\partial q_{k}^{2}}\left\langle q_{k}^{2}\right\rangle,
$$

where the first and second dipole derivatives were calculation $a b$ initio, and the expectation values of the normal coordinates were determined using standard second-order perturbation theory. Further details can be found in X. Zhang, M. R. Nimlos, G. B. Ellison, M. E. Varner and J. F. Stanton, J. Chem. Phys., 124, 084305 (2006) where vibrationally averaged dipole moments of HOONO in the ground and all singly excited vibrational states are documented.

${ }^{25}$ J. Kraitchman, Am. J. Phys. 21, 17 (1953).

${ }^{26}$ W. Gordy and R. Cook, Microwave Molecular Spectra, 3rd ed. (Wiley, New York, 1984).

${ }^{27}$ H. H. Tsai, T. P. Hamilton, J. H. M. Tsai, M. vanderWoerd, J. G. Harrison, M. J. Jablonsky, J. S. Beckman, and W. H. Koppenol, J. Phys. Chem. 100, 15087 (1996).

${ }^{28}$ J. F. Stanton (private communication).

${ }^{29}$ P. Cox and J. M. Riveros, J. Chem. Phys. 42, 3106 (1965).

${ }^{30}$ I. M. Mills, in Molecular Spectroscopy: Modern Research, edited by K. N. Rao and C. W. Matthews (Academic, New York, 1972), p. 115. 